\title{
Abundance Gradient in the Disk of NGC 300
}

\author{
Marija Vlajić ${ }^{1}$, Joss Bland-Hawthorn ${ }^{2}$ and Ken C. Freeman ${ }^{3}$ \\ ${ }^{1}$ Astrophysics, Department of Physics, University of Oxford, Oxford OX1 3RH, UK \\ email: vlajic@astro.ox.ac.uk \\ ${ }^{2}$ Institute of Astronomy, School of Physics, University of Sydney, Australia \\ email: jbh@physics.usyd.edu.au \\ ${ }^{3}$ Research School of Astronomy and Astrophysics, Mount Stromlo Observatory, \\ Australian National University, Australia \\ email: kcf@mso.anu.edu.au
}

\begin{abstract}
The structure of the outer parts of galactic disks and the nature of their stellar populations are fundamental to our understanding of the formation and evolution of spiral galaxies. Ages and metallicity distributions of stars in the outermost regions of spiral disks provide important clues on how and when the disks are assembled. In our earlier work we trace the extended stellar disk of NGC 300 out to a radius of at least 10 disk scale lengths, with no sign of truncation. We now revisit the outer disk of NGC 300 in order to derive the metallicity distribution of the faint stellar population in its outskirts. We find that predominantly old stellar population in the outer disk exhibits a negative abundance gradient - as predicted by the chemical evolution models - out to about $10 \mathrm{kpc}$, followed by the metallicity plateau in the outermost disk.
\end{abstract}

Keywords. galaxies: abundances, galaxies: individual (NGC 300), galaxies: stellar content

\section{Introduction}

Cosmological simulations of galaxy formation have shown that the faint outer regions of disk galaxies are expected to be replete with signatures of the process of galaxy assembly (e.g. Bullock \& Johnston 2005). Due to their long dynamical timescales these regions are able to retain the fossil record from the epoch of galaxy formation in the form of spatial and kinematic distributions, ages and chemical abundances of their stars (Freeman \& Bland-Hawthorn 2002). The structure of the outer parts of galactic disks is therefore central to our understanding of the formation and evolution of disk galaxies.

Until recently, it was believed that all galaxy disks undergo truncation near the Holmberg radius (26.5 mag $\operatorname{arcsec}^{-2}$ ) and that this was telling us something important about the collapsing protocloud of gas that formed the early disk (van der Kruit 1979). This picture was challenged by our earlier finding (Bland-Hawthorn et al. 2005) of a classic exponential disk (Freeman 1970) with no break in NGC 300, out to the distances of $15 \mathrm{kpc}$, corresponding to about 10 disk scale lengths.

The low surface brightness of galactic outskirts presents a challenge for the studies of the outermost regions of galactic disk. The traditional diffuse light imaging is limited at the levels of $\sim 27 \mathrm{mag} \operatorname{arcsec}^{-2}$ by various non-stellar sources of radiation. A more powerful approach is to use the star counts to map out the outskirts of spirals. Only by resolving the outer disk stars can we reach the effective surface brightness levels necessary to trace the outer disk structure (Bland-Hawthorn et al. 2005, Ferguson et al. 2007). 


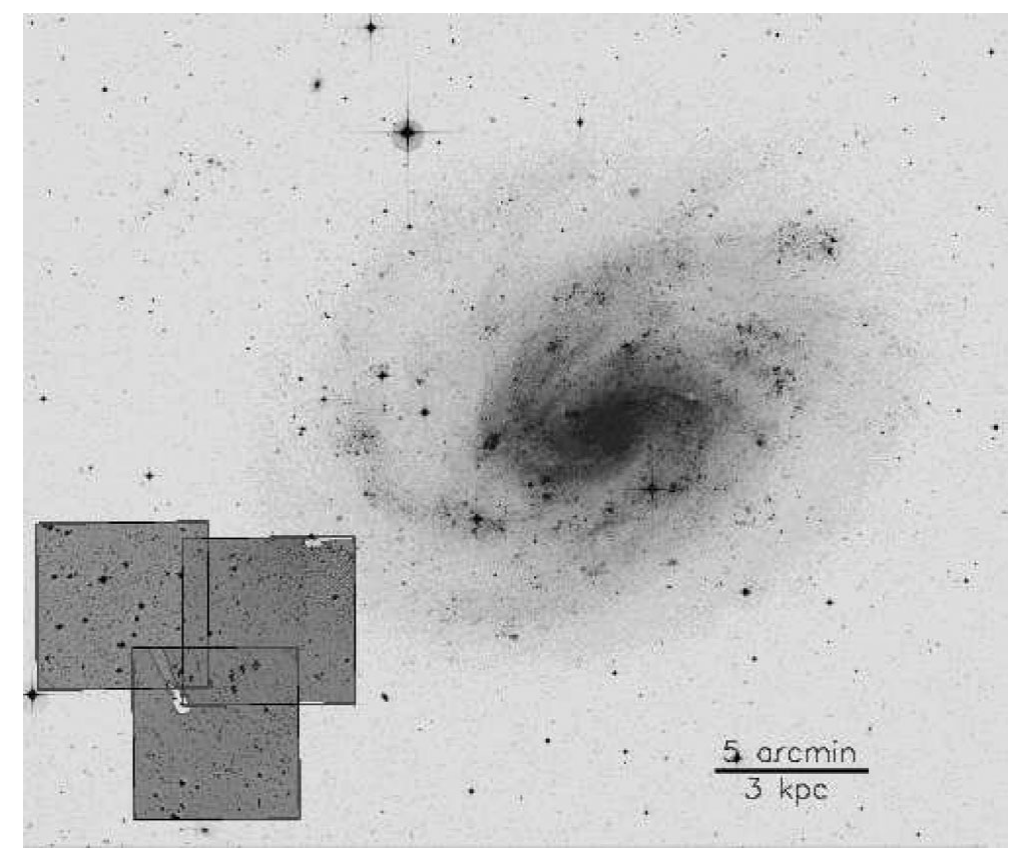

Figure 1. The Digitized Sky Survey (DSS) wide-field image of NGC 300. (North is to the top, east is to the left.) Squares mark the positions of GMOS $5^{\prime} .5 \times 5^{\prime} .5$ field of view.

\section{Observations and Data Analysis}

The deep $g^{\prime}$ and $i^{\prime}$ band images of three fields in the outer disk of NGC 300 were obtained with the Gemini Multi Object Spectrograph (GMOS) on Gemini South telescope. The locations of the fields, spanning the galactocentric radii of $7-16 \mathrm{kpc}$, are shown in Fig. 1. With the total observing time of 34 hours and the average image quality of $0.7^{\prime \prime}$ and $0.6^{\prime \prime}$ FWHM in $g^{\prime}$ and $i^{\prime}$, respectively, we reach the $50 \%$ completeness limit at $26.8-27.4$ in $g^{\prime}$ and $26.8-27.4$ in $i^{\prime}$ band.

The data were reduced using the standard Gemini/IRAF reduction routines and analyzed with the DAOPHOT/ALLSTAR software suite (Stetson 1987). We performed extensive artificial stars tests to assess the data completeness, as well as derive realistic estimates of the photometric errors and criteria for discarding spurious and non-stellar detections. At these faint depths, careful consideration must be given to the contaminating effect of the background galaxy population. We estimated the contribution of unresolved faint galaxies to our star counts using the observations of the William Herschel Deep Field (WHDF; Metcalfe et al. 2001).

\section{Results and Discussion}

In Vlajić et al. (2008) we revisit NGC 300 to investigate the faint stellar population in its outskirts. Top panel of Fig. 2 shows the outer disk surface brightness profile of the galaxy derived from the $i^{\prime}$-band starcounts of the old red giant branch stars. Surface brightness is calculated in $0.5^{\prime}$ annuli by summing the flux of all stars in a given annulus and correcting for the radial completeness, number of pixels in the annulus and the pixel scale to obtain surface brightness in the units of mag $\operatorname{arcsec}^{-2}$. We also correct for the light missed due to the fact that we detect only the brightest stars in the outskirts of NGC 300 and the inclination of the galaxy. The surface brightness profile confirms the 


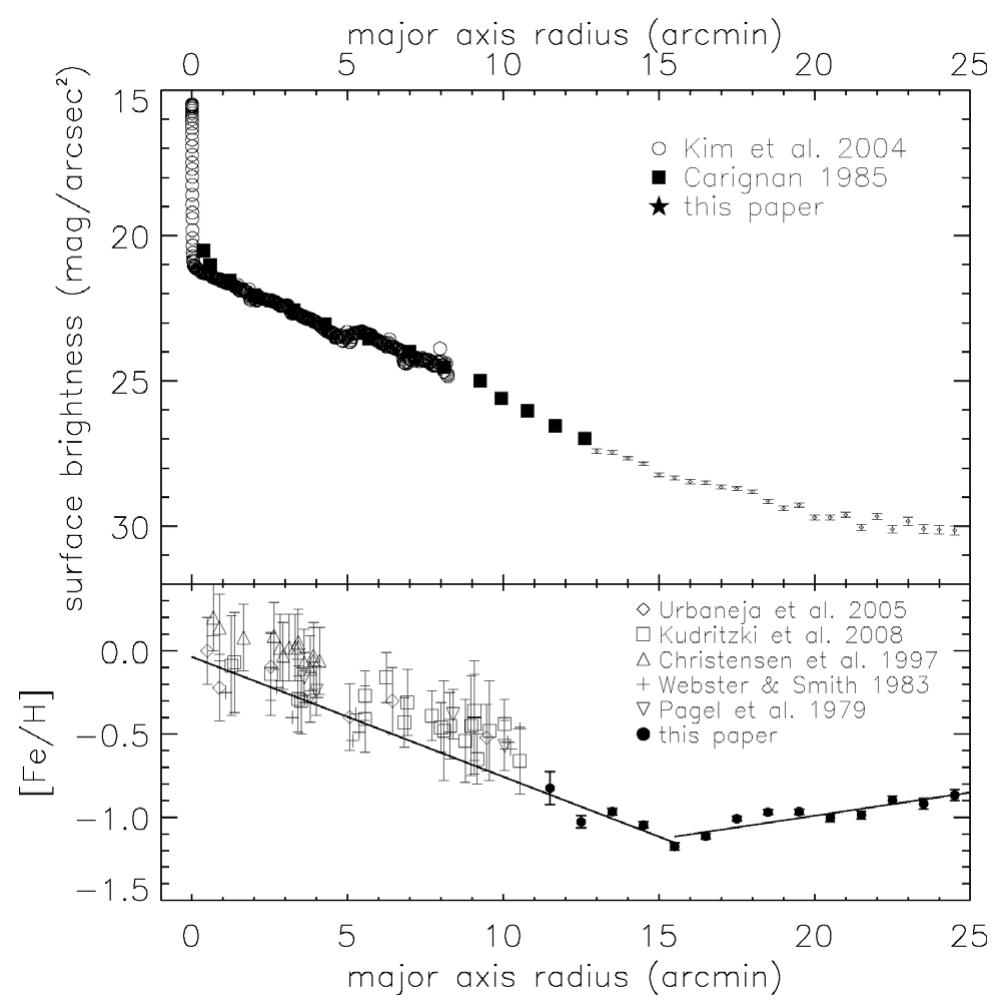

Figure 2. Top: Surface brightness profile of NGC 300. Open circles are I-band surface photometry data from Kim et al. (2004); the data points have been shifted downward by 1.2 mag. Full squares are $\mathrm{B}_{J}$ surface photometry measurements taken from Carignan (1985) and have been shifted upward by $0.4 \mathrm{mag}$. The star symbols are our surface brightness measurements derived from star counts. Bottom: Metallicity gradient in the disk of NGC 300. Inner disk data points are from the spectroscopic abundance studies listed in the figure. Filled circles are outer disk mean metallicities in $1^{\prime}$ bins and solid lines are the linear fits to these values in the regimes $R<15.5^{\prime}$ and $R>15.5^{\prime}$

earlier finding (Bland-Hawthorn et al. 2005) of the extended stellar disk in NGC 300 with the surface brightness declining exponentially out to $\sim 25^{\prime}$, or about 10 disk scale lengths. We note here that only three other disk have been traced down to the same effective surface brightness levels so far - that of the Galaxy(Ibata et al. 2003), M31 (Irwin et al. 2005) and M33 (Ferguson et al. 2007). Except for the case of a "sub-exponential" disk of M33 the other three disks are either pure exponentials or "super-exponential" and are detected down to the effective surface brightness levels below $\sim 30 \mathrm{mag} \operatorname{arcsec}^{-2}$. The existence of stars at these extremely low surface densities $\left(0.01 \mathrm{M}_{\odot} \mathrm{pc}^{-2}\right.$, BlandHawthorn et al. 2005) in the outer galactic disk of NGC 300 is puzzling. More insight about the nature of this faint stellar population can be gained by studying the metallicity distribution of the outer disk stars.

We use the position of old red giant branch stars on the color-magnitude diagram, together with the theoretical stellar evolutionary tracks of VandenBerg et al. (2006), to derive the metallicity distribution function of the stars in the outer disk of NGC 300. The colors of the stars on the red giant branch are more sensitive to metallicity than to age which, despite the fact that there is an inherent age-metallicity degeneracy and that the procedure assumes coeval star formation, allows for the metallicities to be determined without introducing large uncertainties. 
In the bottom panel of Fig. 2 we show the metallicity gradient in the disk of NGC 300 . The metallicities of the outermost $14^{\prime}$ are calculated as mean metallicities of the stars within $1^{\prime}$ wide annuli. We determine the metallicity gradient for stars with $R<15.5^{\prime}$ and $R>15.5^{\prime}$; these linear fits are shows as solid lines in the figure.

Radial abundance profiles represent one of the most important observational constraints for the models of the evolution of spiral galaxies. Abundance gradients arise naturally in the chemical evolution models as a consequence of the radial dependence of yield, star formation and gas infall; observed abundances exhibit slow negative gradient with large, but uniform, dispersion.

In the outer disk of NGC 300 we find that abundance gradient changes slope at a radius of $15.5^{\prime}(\sim 9.3 \mathrm{kpc})$. Does this mark the end of the stellar disk and a transition to an old halo? The argument against the disk-halo transition at $15.5^{\prime}$ is the relatively high metallicity of the outermost field. Typical stellar halos are more metal-poor than what we find in the outer disk of NGC 300. In the Local Group, the halos of the Galaxy (e.g. Carollo et al. 2007) and M31 (Chapman et al. 2006, Koch et al. 2007) have metallicities of the order of $[\mathrm{Fe} / \mathrm{H}]=-1.5$, or lower. Even the less massive, almost bulge-less, M33 which is expected to have properties similar to those of NGC 300 has a metal-poor halo with $[\mathrm{Fe} / \mathrm{H}]=-1.3$ to -1.5 , and a more metal-rich disk with the photometric metallicity of $[\mathrm{Fe} / \mathrm{H}] \approx-0.9$ (McConnachie et al. 2006), similar to the outer disk metallicities we derive. We also note here that stellar halos are usually found to exhibit a negative metallicity gradient (e.g. Koch et al. 2007), which is in disagreement with our positive or flat gradient in the outer disk.

Metallicity trend in which the decline in abundances with radius is followed by a metallicity plateau, similar to what we find in the outer disk of NGC 300, has already been observed in M31 (Worthey et al. 2005) and the Galaxy (Carney et al. 2005, Yong et al. $2005,2006)$. The latter authors also find the $\alpha$-enhanced outer disk with $[\alpha / \mathrm{Fe}] \approx 0.2$. We note here that M33 does not exhibit a flattening of its metallicity gradient (Barker et al. 2007). However, this is not surprising given that M33 experiences a break in its light profile at $\sim 35^{\prime}$ and the surface brightness falls below 31 mag $\operatorname{arcsec}^{-2}$ at the radius of $60^{\prime}$ (Ferguson et al. 2007), where halo abundances start to dominate.

We present two scenarios to explain the metallicity plateau (or upturn) in the outer disks of spirals.

In the radial mixing scenario, the mechanism described by Sellwood \& Binney (2002) is responsible for transporting the stars within the disk while preserving the nearly circular orbits and exponential light profile. Bland-Hawthorn et al. (2005) show that the outer disk of NGC 300 is a high-Q environment, with the value of the Toomre Q parameter of $\sim 5 \pm 2$ and as such is expected to be stable to gravitational perturbations. Scattering of stars by spiral waves (Sellwood \& Preto 2002) could then explain the existence of stars in these diffuse regions where no star formation activity is expected. Potentially, stellar migrations could also explain the plateau or upturn we observe in the metallicity gradient of NGC 300. Roškar et al. (2008) reproduce a similar change in trend of the stellar age with radius. However, scattering over such large distances $(5-8 \mathrm{kpc})$ would require spiral waves much stronger than those considered by Sellwood \& Binney (2002).

The accretion scenario is illustrated in Fig. 3. In this picture, the central spheroid is assembled first in the early Universe $(z>4)$. The first generations of stars that form establish a steep abundance gradient in the spheroid (1), reaching the metallicities of around solar. This is supported by solar metallicities seen in QSOs at high redshifts (Hamann \& Ferland 1999, and references therein) and steep abundance gradients observed in the bulge of the Galaxy (e.g. Zoccali et al. 2008). The disk component is established at $z \sim 2$ (Wolfe et al. 2005). The initial disk is metal-poor and with uniform abundance (2). As 


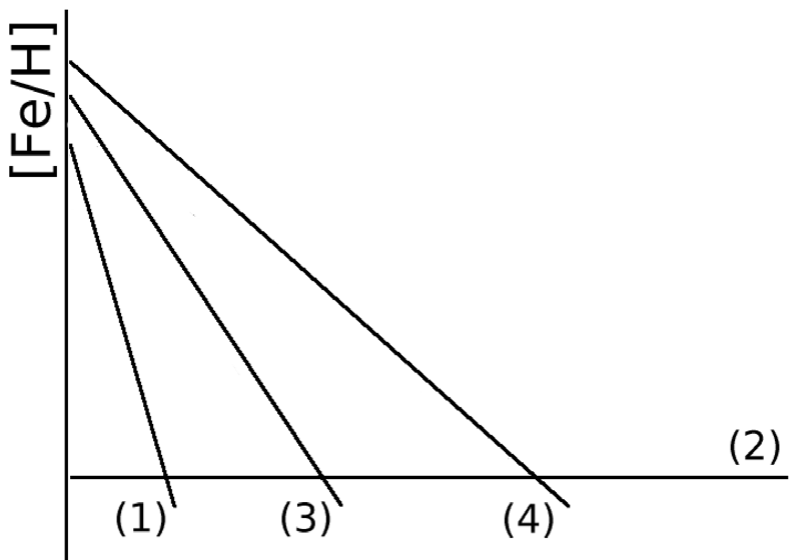

\section{$\mathrm{R}$}

Figure 3. The illustration of the evolution of abundance gradients in disks. (1) Central spheroid is formed at high redshift and intense star formation establishes the steep metallicity gradient. (2) The metal-poor and uniform-metallicity disk is assembled at $z \sim 2$. (3) Star formation sweeps progressively larger radii and enriches the initially low-metallicity regions. (4) As a consequence, the abundance gradient continues to flatten.

the galaxy is built in the inside-out fashion, the extent of star formation migrates towards the outer disk and the parts of the disk that previously had pristine abundances are now forming metals (3). As a consequence, regions of the disk at progressively larger radii become enriched and abundance gradient flattens with time (4). Observations of a variety of chemical gradients in PNe, open clusters, cepheids, OB associations and HII regions (W. Maciel, this volume, and references therein) have shown that older objects exhibit steeper abundance gradients resulting in chemical gradients that become flatter with time. We note however that while some of the chemical evolution models that incorporate inside-out growth of galactic disks were able to reproduce this behavior (e.g. Molla et al. 1997, Hou et al. 2000), others predict steepening of gradient with time (Chiappini et al. 2001). This demonstrates the sensitivity of abundance gradients on adopted form of radial dependence for the star formation and gas infall.

A simple consequence of this picture is that there exist a radius in a galactic disk at which a transition occurs between the star forming disk with a negative abundance gradient and a uniform-abundance initial disk, as observed here in the case of NGC 300, as well as in the disks of the Galaxy (Carney et al. 2005, Yong et al. 2005, 2006) and M31 (Worthey et al. 2005). This is supported by enhanced $[\alpha / \mathrm{Fe}]$ ratios and low $[\mathrm{Fe} / \mathrm{H}]$ both in external gas locally (Collins et al. 2003, 2007) and in the intergalactic medium at high redshift (Prochaska et al. 2003, Dessauges-Zavadsky et al. 2004, 2006).

The accretion scenario suggests that stars today were born roughly in situ, whereas the radial mixing scenario implies that stars largely migrate away from where they were formed. Both of these have consequences for future studies of disk formation.

\section{References}

Barker, M. K., Sarajedini, A., Geisler, D., Harding, P., \& Schommer, R. 2007, AJ, 133, 1125

Bland-Hawthorn, J., Vlajić, M., Freeman, K. C., \& Draine, B. T. 2005, ApJ, 629, 239

Bullock, J. S. \& Johnston, K. V. 2005, ApJ, 635, 931

Carignan, C. 1985, ApJS, 58, 107

Carney, B. W., Yong, D., Teixera de Almeida, M. L., \& Seitzer, P. 2005, AJ, 130, 1111 
Carollo, D., Beers, T. C., Lee, Y. S., Chiba, M., Norris, J. E., Wilhelm, R., Sivarani, T., Marsteller, B., Munn, J. A., Bailer-Jones, C. A. L., Fiorentin, P. R., \& York, D. G. 2007, Nature, 450, 1020

Chapman, S. C., Ibata, R., Lewis, G. F., Ferguson, A. M. N., Irwin, M., McConnachie, A., \& Tanvir, N. 2006, ApJ, 653, 255

Chiappini, C., Matteucci, F., \& Romano, D. 2001, ApJ, 554, 1044

Collins, J. A., Shull, J. M., \& Giroux, M. L. 2003, ApJ, 585, 336

-. 2007, ApJ, 657, 271

Dessauges-Zavadsky, M., Calura, F., Prochaska, J. X., D’Odorico, S., \& Matteucci, F. 2004, $A \mathscr{G} A, 416,79$

Dessauges-Zavadsky, M., Prochaska, J. X., D’Odorico, S., Calura, F., \& Matteucci, F. 2006, $A \& A, 445,93$

Ferguson, A., Irwin, M., Chapman, S., Ibata, R., Lewis, G., \& Tanvir, N. 2007, in: R. S. de Jong (ed.) Island Universes, Structure and Evolution of Disk Galaxies, p. 239

Freeman, K. \& Bland-Hawthorn, J. 2002, ARAA, 40, 487

Freeman, K. C. 1970, ApJ, 160, 811

Hamann, F. \& Ferland, G. 1999, ARAA, 37, 487

Hou, J. L., Prantzos, N., \& Boissier, S. 2000, A\&A, 362, 921

Ibata, R. A., Irwin, M. J., Lewis, G. F., Ferguson, A. M. N., \& Tanvir, N. 2003, MNRAS (Letters), 340, 21

Irwin, M. J., Ferguson, A. M. N., Ibata, R. A., Lewis, G. F., \& Tanvir, N. R., 2005, ApJ (Letters), 628,105

Kim, S. C., Sung, H., Park, H. S., \& Sung, E.-C. 2004, Chinese Journal of Astronomy and Astrophysics, 4, 299

Koch, A., Rich, R. M., Reitzel, D. B., Mori, M., Loh, Y.-S., Ibata, R., Martin, N., Chapman, S. C., Ostheimer, J., Majewski, S. R., \& Grebel, E. K. 2007, Astronomische Nachrichten, 328,653

McConnachie, A. W., Chapman, S. C., Ibata, R. A., Ferguson, A. M. N., Irwin, M. J., Lewis, G. F., Tanvir, N. R., \& Martin, N. 2006, ApJ (Letters), 647, 25

Metcalfe, N., Shanks, T., Campos, A., McCracken, H. J., \& Fong, R. 2001, MNRAS, 323, 795

Molla, M., Ferrini, F., \& Diaz, A. I. 1997, ApJ, 475, 519

Prochaska, J. X., Gawiser, E., Wolfe, A. M., Castro, S., \& Djorgovski, S. G. 2003, ApJ (Letters), 595,9

Roškar, R., Debattista, V. P., Stinson, G. S., Quinn, T. R., Kaufmann, T., \& Wadsley, J. 2008, ApJ (Letters), 675, 65

Sellwood, J. A. \& Binney, J. J. 2002, MNRAS, 336, 785

Sellwood, J. A. \& Preto, M. 2002, in: E. Athanassoula, A. Bosma, \& R. Mujica, (eds.), Astronomical Society of the Pacific Conference Series, Vol. 275, Disks of Galaxies: Kinematics, Dynamics and Perturbations, p. 281

Stetson, P. B. 1987, PASP, 99, 191

van der Kruit, P. C. 1979, A\&A, 38, 15

VandenBerg, D. A., Bergbusch, P. A., \& Dowler, P. D. 2006, ApJS, 162, 375

Vlajić, M., Bland-Hawthorn, J., \& Freeman, K. C. 2008, ApJ submitted

Wolfe, A. M., Gawiser, E., \& Prochaska, J. X. 2005, ARAA, 43, 861

Worthey, G., España, A., MacArthur, L. A., \& Courteau, S. 2005, ApJ, 631, 820

Yong, D., Carney, B. W., \& Teixera de Almeida, M. L. 2005, AJ, 130, 597

Yong, D., Carney, B. W., Teixera de Almeida, M. L., \& Pohl, B. L. 2006, AJ, 131, 2256

Zoccali, M., Hill, V., Lecureur, A., Barbuy, B., Renzini, A., Minniti, D., Gomez, A., \& Ortolani, S. $2008, A \mathscr{E} A, 486,177$ 